\title{
LENKE 5 CURVES: IS THORACIC FUSION REALLY NECESSARY?
}

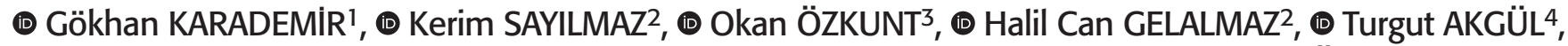 [i]. Murat KORKMAZ5 ${ }^{5}$, iD Fatih DiKiCi², I0 Ünsal DOMANIÇ4}

\author{
${ }^{1}$ Avcılar Murat Kölük State Hospital, Clinics of Orthopaedics and Traumatology, Istanbul, Turkey \\ ${ }^{2}$ Acıbadem University, Atakent Hospital, Department of Orthopaedics and Traumatology, Istanbul, Turkey \\ ${ }^{3}$ Memorial Istanbul Hizmet Hospital, Clinics of Orthopaedics and Traumatology \\ ${ }^{4}$ istanbul University, İstanbul Faculty of Medicine, Department of Orthopaedics and Traumatology, İstanbul, Turkey \\ ${ }^{5}$ Koç University, Faculty of Medicine, Department of Orthopaedics and Traumatology, Istanbul, Turkey
}

\begin{abstract}
Objective: The recommended surgery for Lenke type 5 curve is only the fusion of structural curve (SF: Selective fusion). However, some surgeons prefer to fuse both the thoracolumbar and lumbar (TL/L) curve and the non-structural thoracic (T) curve (NSF: Non-selective fusion). There is a lack of data with regard to the mid-to-long-term outcomes of the SF and NSF. In this study, it was aimed to compare SF and NSF in terms of TL/L and $T$ curve correction rates and the prognosis of the corrected curves.

Materials and Methods: A retrospective study of AIS patients treated at a single institution was conducted. A total of 59 patients (55F/M4) were included in the study. Preoperative, early postoperative and last follow-up TL/L Cobb and T Cobb angles were measured with software. SF and NSF correction rate comparison was done using the Mann-Whitney $U$ test.

Results: Overall, 35 patients underwent NSF while 24 patients underwent SF. The mean follow-up duration was $43 \pm 18.05$ months (54-98). Early T Cobb correction rate was $69 \%$ in group 1 (SF) and $79 \%$ group 2 (NSF); however, this difference was not statistically significant when the groups were compared ( $p=0.71138$ ). Last follow-up T Cobb correction rates for group 1 and group 2 were $66 \%$ and $79 \%$, respectively ( $p=0.5485)$. Early TL/ L Cobb correction rate was $78 \%$ in group 1 and it was $79 \%$ in group 2 ( $p=0.8493)$. Last follow-up TL/L Cobb follow-up correction rates for groups were $79 \%$ and $76 \%$, respectively ( $\mathrm{p}=0.9203)$.

Conclusion: This study concluded that SF had favourable outcomes without loss of correction for the patients with Lenke type 5C AIS in the mid-to long-term.

Keywords: Lenke type 5C AIS, selective fusion, non-selective fusion, loss of correction, prognosis
\end{abstract}

\section{INTRODUCTION}

Lenke type 5 adolescent idiopathic scoliosis (AIS) covers a structural thoracolumbar and lumbar (TL/L) curve and nonstructural proximal or main thoracic $(\mathrm{T})$ curves $^{(1)}$. The widely accepted treatment for Lenke type 5 curves is performing selective fusion (SF) of the structural TL/L curve ${ }^{(1-4)}$. However, some spine surgeons treat these curves non-selectively, while others tend to treat selectively, with the aim of protecting certain mobile segments in the thoracic spine ${ }^{(5,6)}$. The SF technique was reported to be more advantageous in terms of shorter operation time, shorter hospital stay and lower morbidity ${ }^{(7-9)}$. The non-selective fusion (NSF) technique, involving the fusion of both the structural and minor T curves, was reported to have higher correction rates and longer preservation of the corrections, as the main advantages ${ }^{(2,3,9)}$. To the best of our knowledge, studies comparing the outcomes of SF versus NSF treatment of patients with Lenke type 5C AIS are limited and the available data are controversial ${ }^{(10,11)}$. The aim of this study was to present the mid-to long-term radiologic outcomes of the patients with Lenke type 5C AIS who underwent SF and NSF, by evaluating the correction rates and the loss of correction of the TL/L and T curves.

\section{MATERIALS AND METHODS}

This was a retrospective review of 71 patients with Lenke type $5 \mathrm{C}$ AIS who underwent SF versus NSF (Figure 1). All of the patients were operated by one surgeon at a single institution between June 1998 and July 2009. The inclusion criteria were as follows: patients with Lenke type $5 \mathrm{C}$ curve, operated only with the posterior approach, using pedicle screws, having no past history of spine surgeries, together with having standing full-body spine radiographs taken preoperatively, early postoperatively and during the last follow-up visit. Patients with a follow-up

Address for Correspondence: Gökhan Karademir, Avcılar Murat Kölük State Hospital, Clinics of Orthopaedics and Traumatology, İstanbul, Turkey E-mail: dr@gokhankarademir.com Received: 30.04.2020 Accepted: 11.05.2020

ORCID ID: orcid.org/0000-0002-0894-9785 
period of at least 2 years were included in the study. Patients who had previous surgery, hybrid constructs, anterior surgery, spinal osteotomies and patients whose radiographs did not meet the required standards were excluded from the study. Therefore, twelve patients who did not meet the inclusion criteria were excluded.

Fifty-nine patients (55 females, four males) with a mean age of $16.7 \pm 3.8$ and a mean follow-up period of $43 \pm 18.05$ months, in concordance with the inclusion criteria were included in the study. The choice of treatment was basically determined according to two criteria: (1) The presence of a thoracic hump upon examination and the need to perform a long-level fusion to handle it. (2) The aim of a better correction of the trunk shift, with a long-level fusion. Thus, patients were divided into two groups: Group 1 was composed of 24 patients ( 22 females/two males) who underwent SF and group 2 was composed of 35 patients (33 females/two males) who underwent NSF (Table 1). The surgeries were performed by the same experienced spinal surgeon on the basis of his personal experiences according to the clinical examination and the posteroanterior (PA) and lateral side-bending radiographs prior to surgery. All the patients were operated under general anaesthesia and placed in a prone position on a surgical table. All the patients also underwent

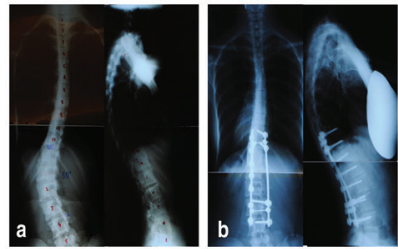

Selective fusion

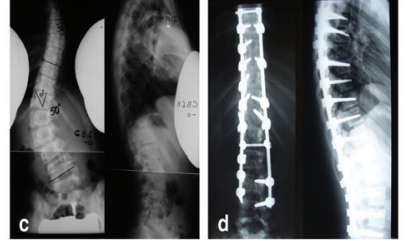

Non-selective fusion
Figure 1. (a) Preoperative and (b) Postoperative radiography of a 16-year -old female patient with a $50^{\circ} \mathrm{TL} / \mathrm{L}$ Cobb angle corrected by selective fusion (c) Preoperative and (d) Postoperative radiography of an 11-year-old female patient with a $50^{\circ} \mathrm{TL} / \mathrm{L}$ Cobb angle corrected by non-selective fusion posterior instrumentation and fusion. The pedicle screw rod system was used for fixation. Several surgical manoeuvres were used such as apical vertebral de-rotation, rod rotation, convex compression and concave distraction.

The study was performed by the ethical standards of the 1964 Declaration of Helsinki (ethics committee permission number 2017/17-3). Oral and written informed consent was obtained to publish the data.

\section{Statistical Analysis}

Radiologic evaluations were based on standing fullbody radiographs that were taken preoperatively, early postoperatively (first week) and at the latest follow-up visit by a researcher who did not participate in the surgeries. TL/L Cobb angle and T Cobb angle were measured. Radiographic measurements were done using the Surgimap software (New York, NY, USA). Kolmogorov-Smirnov test was used to assess the distribution of the data, while the Mann-Whitney $U$ test was used to compare the outcomes. Statistical significance was defined as $p<0.05$ (SPSS 24.0, IL, USA).

\section{RESULTS}

In patients with SF (group 1), the mean TL/L Cobb and T Cobb angles in the preoperative, early postoperative periods and at the last follow-up visit were $39.68^{\circ} \pm 9^{\circ}$ and $16.5^{\circ} \pm 6.7^{\circ}$; $8.7^{\circ} \pm 8.2^{\circ}$ and $5.3^{\circ} \pm 5^{\circ}$; and $9.5^{\circ} \pm 8.4^{\circ}$ and $6^{\circ} \pm 4.9^{\circ}$, respectively (Table 2). Comparing the preoperative and early postoperative data, we found that the spontaneous postoperative correction of the T curves was statistically significant $(p<0.05)$, while the loss of correction observed during the follow-up period was found not to be statistically significant ( $p>0.05)$. In patients with NSF (group 2), the mean TL/L Cobb and T Cobb angles in the preoperative, and early postoperative periods were $43.6^{\circ} \pm 8.4^{\circ}$ and $25.2^{\circ} \pm 9.5^{\circ}$; and $8.4^{\circ} \pm 6.6^{\circ}$ and $5.3^{\circ} \pm 5.6^{\circ}$, respectively. At the last follow-up visit, the mean TL/L Cobb angle was $8.9^{\circ}$ and $\mathrm{T}$ Cobb angle was $5.45^{\circ}$ (Table 2).

Table 1. Patients' demographic characteristics

\begin{tabular}{llll}
\hline & Group 1 (SF) (24 patients) & Group 2 (NSF) (35 patients) & $p$ value \\
\hline Gender & $22 \mathrm{~F}, 2 \mathrm{M}$ & $33 \mathrm{~F}, 2 \mathrm{M}$ & 1.00 \\
\hline Mean age at surgery (year) & 16.5 (range, 14-21) & 17 (range, 11-26) & 0.594 \\
\hline Follow-up duration (month) & 40 (range, 24-77) & 45 (range, 24-98) & 0.105 \\
\hline SF: Selective fusion, NSF: Non-selective fusion, F: Female, M: Male & &
\end{tabular}

Table 2. Radiographical outcome measures at all study interval assessments

\begin{tabular}{lllllllllll}
\hline Variables & & \multicolumn{3}{c}{ Pre-operative } & \multicolumn{3}{c}{ Early post-operative } & \multicolumn{2}{c}{ Last follow-up } \\
& & Group 1 & Group 2 & $p$ value & Group 1 & Group 2 & $p$ value & Group 1 & Group 2 & $p$ value \\
\hline $\begin{array}{l}\text { TL/L Cobb Angle } \\
\text { (degree) }\end{array}$ & Min-max & $27-61$ & $27-66$ & 0.081 & $0-36$ & $1-33$ & 0.9703 & $1-36$ & $1-32$ & 0.767 \\
\hline $\begin{array}{l}\text { T Cobb Angle } \\
\text { (degree) }\end{array}$ & Mean \pm SD & $40 \pm 9$ & $44 \pm 8$ & & $9 \pm 8$ & $8 \pm 7$ & & $9 \pm 6$ & 0.767 \\
\hline
\end{tabular}

TL/L: Thoracolumbar/Lumbar, T: Thoracic, Min: Minimum, Max: Maximum, SD: Standard deviation 
turkishspine

Although the early $T$ Cobb correction rate seemed a little lower in group 1 (69\%) when compared to group 2 (79\%), this difference was not statistically significant $(p=0.71138)$. The $T$ Cobb correction rates at the last follow-up were $66 \%$ and $79 \%$, respectively $(p=0.5485)$ (Table 3$)$.

Early TL/L Cobb correction rate was 78\% in group 1 and $79 \%$ in group 2 ( $p=0.8493)$. The TL/L Cobb correction rates at the last follow-up were $79 \%$ and $76 \%$, respectively $(p=0.9203)$ (Table 4$)$.

\section{DISCUSSION}

The main goal of surgery in patients with Lenke type 5 AIS is to prevent progression of the curve and to correct the deformity with minimum fusion levels, in order to provide a mobile and functional spine ${ }^{(1,5,7)}$. In line with the recommendations of the Lenke classification, many surgeons prefer SF in the treatment of patients with Lenke type $5 \mathrm{AIS}^{(10,11)}$. However, in many studies, it has been reported that a considerable number of experienced spine surgeons prefer NSF for this group of patients, in up to $17 \%$ of cases $^{(9-11)}$. That could be because they believe that adequate correction cannot be achieved in minor $T$ curve with $\mathrm{SF}^{(5)}$ or that the prognosis of the correction obtained is uncertain ${ }^{(3)}$. On the other hand, in a study conducted by Zhang et al. ${ }^{(5)}$, it has been reported that the TL/L curve correction rate was $84.9 \%$ and the spontaneous T curve correction was $48.5 \%$ in patients with Lenke type $5 \mathrm{AIS}$, while no significant increase with regard to the loss of correction was detected during the long-term follow-up. Similar to Zhang et al.(5)'s findings, Senkoylu et al.(3) reported that the spontaneous correction in $T$ curves was satisfactory and there was no loss of correction at a mean follow-up of 4 years. Also, correction loss was not found to be statistically significant in patients with both SF and NSF at long-term follow-up by Sanders et al. ${ }^{(8)}$. In our study, the correction rate, which was $68 \%$ in the early period for $\mathrm{T}$ Cobb and $66 \%$ at a mean follow-up of 42.3 months, agrees with the findings of the aforementioned studies.
In contrast, Zang et al.(5) reported a loss of correction in 22 of 45 patients with Lenke type 5C AIS at a mean follow-up of 36 months. The authors defined higher flexibility and better immediate correction as risk factors for the loss of correction.

Sanders et al..$^{(8)}$ reported a TL/L:T Cobb ratio $>1.25$; might be the best predictor with regard to the correction of the minor curve when combined with the degree of T curve, while reporting that a ratio close to 1 could lead to progression of the T curve. In our study, the TL/L:T Cobb ratio was 2.4 and favourable outcomes were obtained with a rate of $69 \%$ in the correction of minor T curve in the SF group. The lower angle in the T curve was found to be an important factor in fixing the frontal plane deformity, while a T Cobb angle greater than $60^{\circ}$ was shown to make the spontaneous regression impossible ${ }^{(8)}$. None of the patients in this study had a $\mathrm{T}$ Cobb angle greater than $60^{\circ}$. It was reported that the $T L / L$ Cobb angle had an important influence on the surgical success. Although structural TL/L curves with higher angles were reported to be more difficult to correct, full correction of the major TL/L Cobb was also reported to be the main factor for the spontaneous correction of a minor $\mathrm{T}$ curve ${ }^{(8)}$. The highest angle of TL/L Cobb was $53^{\circ}$ in this study and we detected no significant difference with regard to the loss of correction during the follow-up; even with the high TL/L curve angles.

On the other hand, it was observed that there is only one study comparing SF and NSF in patients with Lenke type 5 AIS in the literature. Lark et al. ${ }^{(6)}$ stated that the T Cobb angle improved significantly in both groups; however, the degree of improvement was significantly higher in the NSF group at the 2 -year follow-up of 150 patients. Contrary to previous reports, the relatively higher correction rates obtained in the NSF group was not statistically significant in our study. The authors stated that the shortcoming of that study was a non-standardised surgical procedure (anterior or posterior) and a short follow-up period of 2 years. The mean follow-up of 42.3 months,

Table 3. Statistical comparison of the T Cobb correction rate in the early postoperative period and at the last follow-up

\begin{tabular}{lllll}
\hline Variables & & Group 1 $(\mathrm{n}=24)$ & Group $2(\mathrm{n}=35)$ & $p$ and $z$ value \\
\hline \multirow{2}{*}{ Early correction rate (\%) } & Min-max & $31-100$ & $40-100$ & $\mathrm{z}=0.3716$ \\
& Mean \pm SD & $69 \pm 25$ & $79 \pm 21$ & $\mathrm{p}=0.7113$ \\
\hline \multirow{2}{*}{ Last follow-up correction rate (\%) } & Min-max & $31-100$ & $40-100$ & $\mathrm{z}=59656$ \\
& Mean \pm SD & $66 \pm 24$ & $79 \pm 21$ & $\mathrm{p}=0.5485$ \\
\hline
\end{tabular}

z: Mann-Whitney U statistic, T: Thoracic, Min: Minimum, Max: Maximum, SD: Standard deviation, n: Number

Table 4. Statistical comparison of the TL/L Cobb correction rate in the early postoperative period and at the last follow-up

\begin{tabular}{lllll|}
\hline Variables & & Group 1 $(\mathrm{n}=24)$ & Group 2 $(\mathrm{n}=35)$ & $p$ and $z$ value \\
\hline \multirow{2}{*}{ Early correction rate (\%) } & Min-max & $50-100$ & $38-98$ & $\mathrm{z}=0.1906$ \\
& Mean \pm SD & $78 \pm 36$ & $80 \pm 38$ & $\mathrm{p}=0.8493$ \\
\hline \multirow{2}{*}{ Last follow-up correction rate (\%) } & Min-max & $50-96$ & $63-100$ & $\mathrm{z}=-0.0991$ \\
& Mean \pm SD & $76 \pm 36$ & $79 \pm 36$ & $\mathrm{p}=0.9203$ \\
\hline
\end{tabular}

z: Mann-Whitney U statistic, TL/L: Thoracolumbar/Lumbar, Min: Minimum, Max: Maximum, SD: Standard deviation, n: Number 
standardised posterior approach and evaluating loss of correction can be other strengths of our study.

\section{Study Limitations}

This study was retrospective in nature and contains similar limitations like other retrospective studies. In addition, outcomes of SF and NSF were evaluated only radiologically. The decision to perform a SF versus NSF was based on the surgeon's preference rather than randomisation. However, this potential study bias was minimised by the preoperative similarity in terms of age, gender, follow-up duration, preoperative TL/L Cobb and T Cobb of both groups being compared. The patients in this study were homogeneous and underwent posterior instrumentation and fusion performed by the same surgeon, with the same pedicle screw instrumentation system.

\section{CONCLUSION}

The findings of this study suggest that NSF is not superior to SF in the correction of coronal plane deformities in patients with Lenke type 5C AIS. Moreover, it was found that there was no loss of correction, which was obtained in T minor curves in patients who underwent SF. However, it should be noted that if SF is to be performed, the angle of the major curve together with the T minor curve, TL/L:T Cobb angle ratio, and higher flexibility would be the major determinants of radiological outcomes, predicting the prognosis.

\section{Ethics}

Ethics Committee Approval: The study was performed by the ethical standards of the 1964 Declaration of Helsinki (Acıbadem University and Acıbadem Health Institutions Medical Research Ethics Committee permission number 2017/17-3).

Informed Consent: Oral and written informed consent was obtained to publish the data.

\section{Authorship Contributions}

Surgical and Medical Practice: Ü.D., Concept: F.D., G.K., Design: G.K., K.S., Data Collection or Processing: G.K., O.Ö., Analysis or Interpretation: G.K., H.C.G., M.K., Literature Search: G.K., Writing: G.K., Critical Review: T.A.

Conflict of Interest: No conflict of interest was declared by the authors.
Financial Disclosure: The authors declared that this study received no financial support.

\section{REFERENCES}

1. Lenke LG, Edwards CC, Bridwell KH. The Lenke classification of adolescent idiopathic scoliosis: how it organizes curve patterns as a template to perform selective fusions of the spine. Spine. (Phila Pa 1976). 2003;28:199-207.

2. Wang F, Xu XM, Wei XZ, Zhu XD, Li M. Spontaneous thoracic curve correction after selective posterior fusion of thoracolumbar/ lumbar curves in Lenke 5C adolescent idiopathic scoliosis. Medicine. 2015;94:e1155.

3. Senkoylu A, Luk KD, Wong YW, Cheung KM. Prognosis of spontaneous thoracic curve correction after the selective anterior fusion of thoracolumbar/lumbar (Lenke 5C) curves in idiopathic scoliosis. The Spine Journal. 2014;14:1117-24.

4. Sun Z, Qiu G, Zhao Y, Wang Y, Zhang J, Shen J. Lowest instrumented vertebrae selection for selective posterior fusion of moderate thoracolumbar/lumbar idiopathic scoliosis: lower-end vertebra or lower-end vertebra+1? European Spine Journal. 2014;23:1251-7.

5. Zhang Y, Lin G, Wang S, Zhang J, Shen J, Wang Y, Guo J, Yang X, Zhao L. Higher flexibility and better immediate spontaneous correction may not gain better results for nonstructural thoracic curve in Lenke $5 \mathrm{C}$ AIS patients: risk factors for its correction loss. Spine. 2016;41:1731.

6. Lark RK, Yaszay B, Bastrom TP, Newton PO, Harms Study Group. Adding thoracic fusion levels in Lenke 5 curves: risks and benefits. Spine. 2013;38:195-200.

7. Edwards CC, Lenke LG, Peelle M, Sides B, Rinella A, Bridwell KH. Selective thoracic fusion for adolescent idiopathic scoliosis with $C$ modifier lumbar curves: 2-to 16-year radiographic and clinical results. Spine. 2004;29:536-46.

8. Sanders $A E$, Baumann $R$, Brown $H$, Johnston $C E$, Lenke $L G$, Sink E. Selective anterior fusion of thoracolumbar/lumbar curves in adolescents: when can the associated thoracic curve be left unfused? Spine. 2003;28:706-13.

9. Crawford III CH, Lenke LG, Sucato DJ, Richards III BS, Emans JB, Vitale $M G$, et al. Selective thoracic fusion in Lenke 1C curves: prevalence and criteria. Spine. 2013;38:1380-5.

10. Clements DH, Marks M, Newton PO, Betz RR, Lenke L, Shufflebarger $\mathrm{H}$, et al. Did the Lenke classification change scoliosis treatment? Spine. 2011;36:1142-5.

11. Puno RM, An KC, Puno RL, Jacob A, Chung SS. Treatment recommendations for idiopathic scoliosis: an assessment of the Lenke classification. Spine. 2003;28:2102-14. 